\title{
"Do It Yourself" Microbial Cultivation Techniques for Synthetic and Systems Biology: Cheap, Fun, and Flexible
}

OPEN ACCESS

Edited by:

George Tsiamis,

University of Patras, Greece

Reviewed by:

Panagiota Stathopoulou,

University of Patras, Greece Francisco Solano,

Universidad de Murcia, Spain

${ }^{*}$ Correspondence:

Ya-Tang Yang

ytyang@ee.nthu.edu.tw

Specialty section:

This article was submitted to Systems Microbiology,

a section of the journal

Frontiers in Microbiology

Received: 15 February 2018 Accepted: 04 July 2018

Published: 30 July 2018

Citation:

Pilizota T and Yang Y-T (2018) "Do It

Yourself" Microbial Cultivation

Techniques for Synthetic

and Systems Biology: Cheap, Fun,

and Flexible. Front. Microbiol. 9:1666.

doi: 10.3389/fmicb.2018.01666

\author{
Teuta Pilizota ${ }^{1}$ and Ya-Tang Yang ${ }^{2 *}$ \\ ${ }^{1}$ Centre for Synthetic and Systems Biology, School of Biological Sciences, University of Edinburgh, Edinburgh, \\ United Kingdom, ${ }^{2}$ Department of Electrical Engineering, National Tsing Hua University, Hsinchu, Taiwan
}

With the emergence of inexpensive 3D printing technology, open-source platforms for electronic prototyping and single-board computers, "Do it Yourself" (DIY) approaches to the cultivation of microbial cultures are becoming more feasible, user-friendly, and thus wider spread. In this perspective, we survey some of these approaches, as well as addon solutions to commercial instruments for synthetic and system biology applications. We discuss different cultivation designs, including capabilities and limitations. Our intention is to encourage the reader to consider the DIY solutions. Overall, custom cultivation devices offer controlled growth environments with in-line monitoring of, for example, optical density, fluorescence, $\mathrm{pH}$, and dissolved oxygen, all at affordable prices. Moreover, they offer a great degree of flexibility for different applications and requirements and are fun to design and construct. We include several illustrative examples, such as gaining optogenetic control and adaptive laboratory evolution experiments.

Keywords: bioreactor, synthetic biology, systems biology, microbial cultivation, evolution, optogenetics

\section{INTRODUCTION}

Arguably, microbiology is experiencing a renaissance driven by several applied and basic research fields, e.g., combating antimicrobial resistance and using microbes in industrial biotechnology relies on successes of systems and synthetic biology that strive to understand and engineer microorganisms. At the basis of these research efforts is the ability to culture microorganisms with sufficient flexibility and throughput. For example, synthetic biology is recognized as an emerging technology able to combine research excellence with the power of businesses to develop novel products to drive economic growth (Pleiss, 2006). The vision rests on the ability to offer enhanced control of gene expression and metabolic pathways, either through modulating existing pathways or by introducing novel pathways in a given organism (Pleiss, 2006). Thus, the engineered microorganisms can effectively become factories, sustainably producing a range of products, for example, biologics and diagnostics (Scognamiglio et al., 2015). The process of strain engineering passes through a design-build-test-learn iterative cycle, which, at the start, is high throughput but involves working with small culture volumes that can later be scaled up for commercial and industrial purposes. Similarly, to decipher complex interactions of biological systems and identify mathematical models that best depict their behavior, systems biology benefits from large datasets, 
which are often captured working with small culture volumes that require experiment-specific control of growth environments. Thus, small volumes at the start that can later be scaled up, very specific environmental control of growing cultures, and an increasing range of microbes that are of great interest, make it almost impossible to purchase a microbial cultivation unit that will "do it all." However, inexpensive 3D printing technology and modular open-source platforms for electronic prototyping offer affordable and straightforward building blocks that can expand how we culture microbes, either by offering inexpensive alternatives to commercial products or novel, commercially unavailable, solutions. We survey some of the examples with the intention of encouraging the reader to consider "Do-It-Yourself" (DIY) approaches. We focus on small-volume cultivation (ranging from $100 \mu \mathrm{l}$ to sub-liter scale), simply because it is frequently used in synthetic and systems microbiology. However, DIY approaches are applicable for medium to large culture volumes as well. We also note that microfluidic-based cultivation technology has the potential to further reduce culture volumes and increase throughput, and it is increasingly developed (Ferry et al., 2011; Han et al., 2013; Hegab et al., 2013; Yang and Wang, 2016).

\section{SMALL-VOLUME CULTIVATION DESIGN CONSIDERATIONS}

Arguably, two most frequent small-volume microbial cultivation units are a bioreactor and a plate-reader. A bioreactor is a vessel that allows controlled growth of a microbial culture, for example, through the supply of fresh media and fixed environmental oxygen levels. Typical bioreactors come with a stirring unit to mix the cells in the culture and achieve full oxygenation of the medium, as well as with in-line monitoring capabilities, e.g., turbidity measurements. Bioreactors are operated in the so-called "fed-batch" or "chemostat" mode (Novick and Szilard, 1950). In the fed-batch mode, the cells are given a fixed amount of nutrients and allowed to grow until nutrients are depleted. In the chemostat mode, fresh medium is constantly supplied while the cells are removed at a constant rate so that a biomass steady state is reached. The microplate or microtiter plate readers are also widely used for gene expression measurement and strain and medium optimization (Zaslaver et al., 2009). Microplates contain a standard number of wells $(24,48,96$, or 384$)$; typically, each well contains few $100 \mu$ l.

Both bioreactors and microplate readers are commercially available, but as the cost of DIY electronic components and 3D printing technology progressively decreases, development of DIY bioreactors, microplate readers, and add-ons to commercial instruments is becoming feasible. Arguably, problems that can benefit most from DIY culturing technology require a great degree of flexibility or levels of control that are not readily available commercially. To give the reader ballpark numbers, $3 \mathrm{D}$ printers now come at $\$ 1500$ USD and plastic consumables are on the order of $2 \mathrm{D}$ printing consumables. Arduino and Raspberry Pi microcontrollers range from $\$ 10$ to $\$ 50$. Lego Mindstorms Robots cost $\sim \$ 300$ and come with a controller box and some Lego parts included. To give a rough estimate of total cost, a previously reported optogenetic bioreactor came under \$300 (Wang and Yang, 2017), and an adaptive laboratory evolution robot at roughly $\$ 2500$ (Arias-Castro, 2017). Below, we discuss examples of custom build bioreactors (Toprak et al., 2013; Takahashi et al., 2014; Wang and Yang, 2017) (Figures 1a-c) and microplate readers (Chen et al., 2012; Heo et al., 2015; Richter et al., 2015) (Figures 2a-c), all of which needed to satisfy few common requirements.

\section{Measuring Cell Number}

There are several typical challenges that most reported examples of DIY bioreactors and microplate readers need to overcome or take into account (Klöckner and Büchs, 2012). First is the implementation of turbidity measurements of a microbial culture, i.e., optical density (OD) measurements. Measuring OD is the most standard way of assessing the growth of microbial cells in a culture, and OD is assumed to be proportional to the number of cells in the culture.

The OD density measurement is traditionally done with a light source and a photodetector. The assumption of linearity holds true in the so-called "single-scattering" regime, i.e., when the number of microbial cells in the culture is sufficiently low for the light to scatter off each cell only once as it passes through the sample. At higher cell culture densities, the socalled "multiple scattering" regime (usually above $10^{8} \mathrm{ml}^{-1}$ cell concentration), OD measurements are no longer a good indication of the cell number and alternatives methods, such as direct counting or the so-called cell growth quantifier, should be used (Koch, 1970; Bruder et al., 2016; Stevenson et al., 2016). The limitation of the OD measurements is particularly relevant for fed-batch bioreactors, microplate readers, or applications where cell size changes, and changes in the index of refraction of the medium or cells are expected (Stevenson et al., 2016). The recently reported cell growth quantifier offers the possibility of extending the cell concentration measurements into the multiple-scattering regime by detecting the backscattered light rather than the transmitted (Schmidt-Hager et al., 2014; Bruder et al., 2016). Similar can be achieved by detecting Escherichia coli's auto-fluorescence, linked with the secretion of flavins (Mihalcescu et al., 2015). Flavins are auto-fluorescent in the green region of the visible spectrum (peaking at $\sim 530 \mathrm{~nm}$ ) and the time derivative of the auto-fluorescence scales linearly with a bacterial concentration in the $\sim 6 \cdot 10^{6}$ to $10^{9} \mathrm{ml}^{-1}$ range. Thus, when not working with cells expressing green fluorescent proteins, monitoring flavin auto-fluorescence extend the concentration ranges of OD measurements (Mihalcescu et al., 2015). On the other end of the scale, microbial cultures of very low densities will scatter very little light and the detection is limited by signal-to-noise ratio. An alternative approach to estimating cell number in low-density microbial cultures is based on bioluminescence photon counting (Kishony and Leibler, 2003). Naturally occurring luminescent bacteria are rare, but the biochemistry and genetics of bioluminescent have been sufficiently characterized to enable transferring optimal combination of genes into Gram-negative bacteria (Winson et al., 1998). When done, resulting bioluminescent intensity 

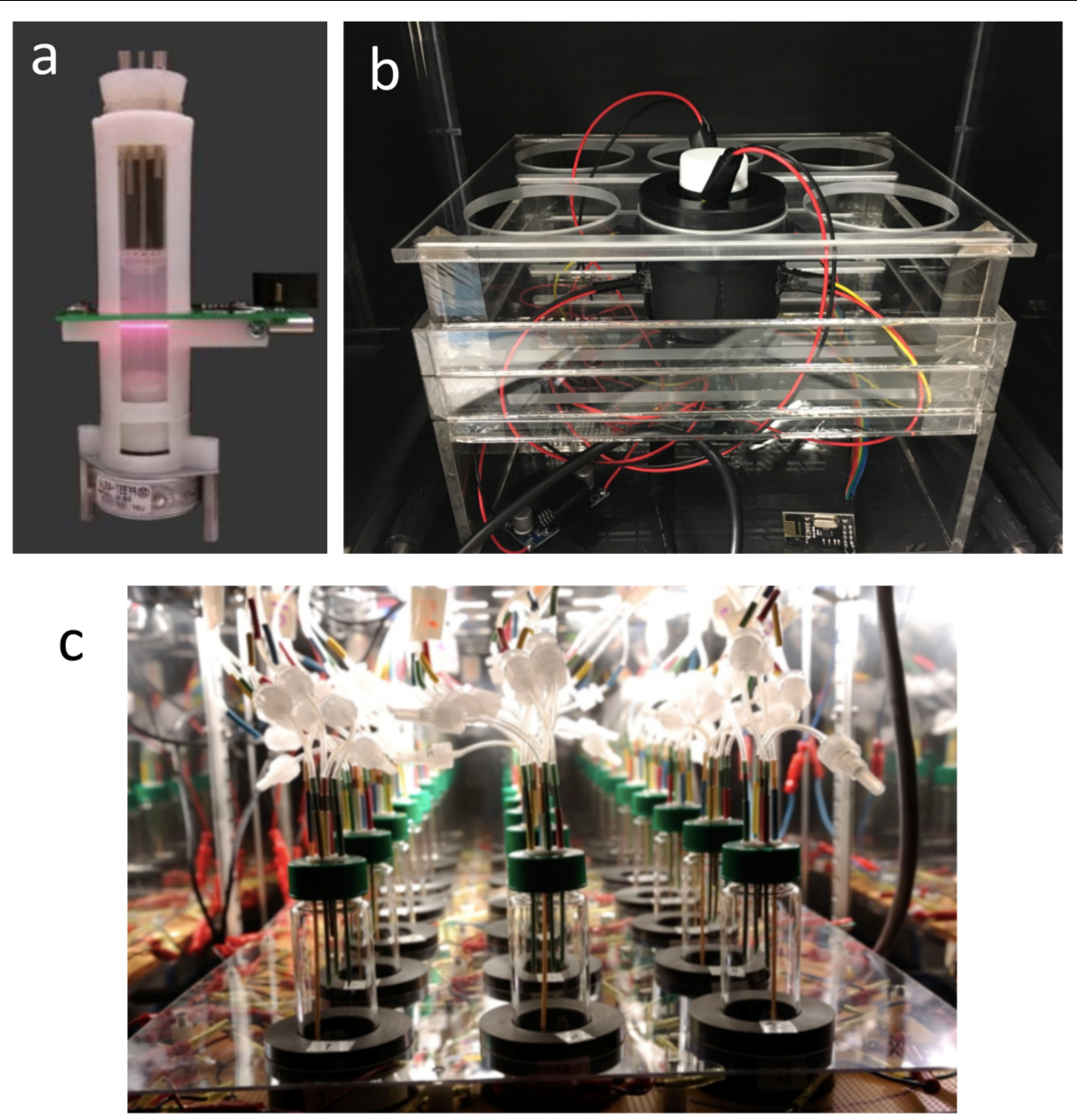

FIGURE 1 | Custom bioreactors for synthetic and system biology. (a) "Turbidostat" built from the 3D printed holder with a laser diode and photodetector installed for optical density measurement (Takahashi et al., 2014). The culture vial is connected to a 3D printed syringe pump to enact the turbidostat mode. (CAmerican Society of Chemistry. Reproduced with permission. (b) Optogenetic mini photo bioreactor (Wang and Yang, 2017). The device integrates light source for control of gene expression and optical density and fluorescence detection for monitoring microbial growth and gene expression. (C) American Society of Chemistry. Reproduced with permission. (c) Morbidostat built for adaptive laboratory evolution (Toprak et al., 2012). The device integrates optical density measurements for monitoring bacterial cell concentration and implements a feedback algorithm to adjust the drug concentration delivered via the tubing connected to the vessel from the top (Figure $\mathbf{3 C}$ gives the feedback scheme). (C) Nature Publishing Group. Reproduced with permission.

of a bacterial culture is linearly proportional to bacterial cell concentration in the range from $\sim 10^{4}$ to $10^{8} \mathrm{ml}^{-1}$, in comparison to OD measurements that are usually within $\sim 10^{7}-$ $10^{8} \mathrm{ml}^{-1}$ cell concentrations (Jepson, 2014; Stevenson et al., 2016). The high accuracy of bacterial cell density measurements achieved with the bioluminescence method enabled, for example, functional classification of antimicrobial drugs according to their pairwise interactions (Yeh et al., 2006).

\section{Achieving Sufficient Oxygen Transfer Rate}

We next discuss the importance of aeration for optimal aerobic growth. The problem can be summarized as the difference between the rate of oxygen consumption by microbes and the rate at which oxygen can be dissolved in the culture media [oxygen transfer rate (OTR)]. Similar to commercial bioreactors, to achieve fully aerobic growth, the DIY cultivation solutions need to ensure oxygen is dissolved faster than microbial cells consume it, which often means achieving a sufficiently large surface area of the culture media (Klöckner and Büchs, 2012). While OTR can be estimated (Hermann et al., 2003; Klöckner and Büchs, 2012) and several solutions to ensuring it is sufficiently high exist (Betts and Baganz, 2006), these most commonly rely on shaking rather than stirring (Klöckner and Büchs, 2012). DIY cultivation devices still predominantly stir, simply because it is technically easier and more cost-effective. Several solutions that do not require shaking could be attractive for DIY cultivation technology, for example, the introduction of baffles to the culture vessel (Gupta and Rao, 2003), or addition of immiscible oxygenated oils (Sklodowska and Jakiela, 2017). Irrespective of the solution, the specific DIY bioreactor should be calibrated to ensure fully aerobic growth in a chosen media by comparing the growth rates achieved in the bioreactor with those in fully oxygenated shaken flasks. 


\section{a}
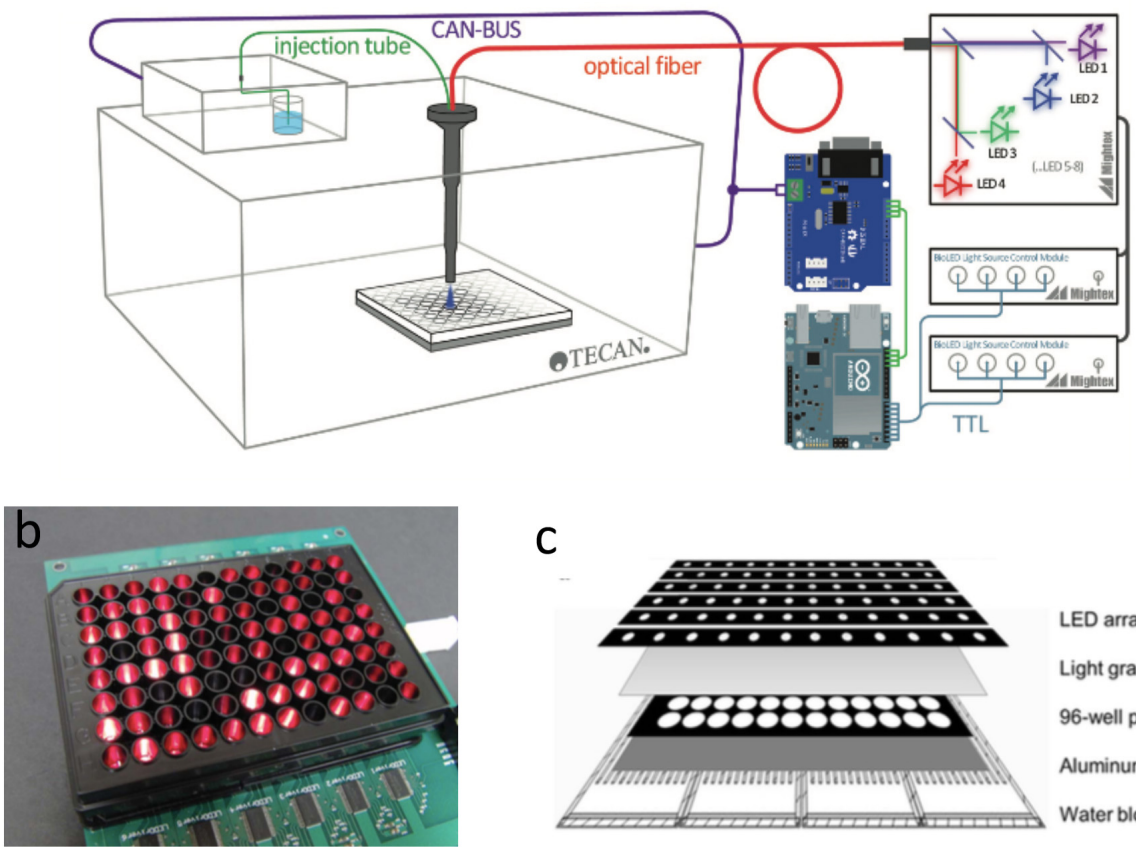

C

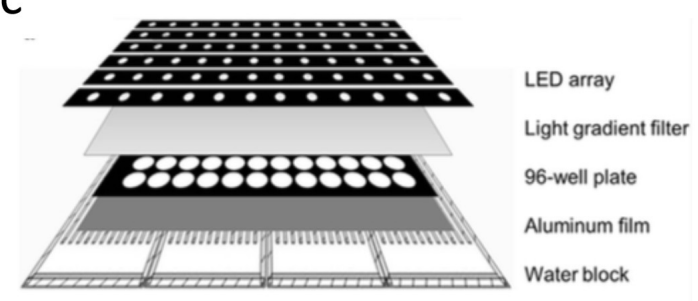

FIGURE 2 | Microplate photobioreactors. (a) Add-on illumination path for a Tecan plate reader (Richter et al., 2015). ๑) Royal Society of Chemistry. Reproduced with permission. (b) An assembled optical microplate for microalgae cultivation (Chen et al., 2012). () Royal Society of Chemistry. Reproduced with permission. (c) Layered assembly for the "Photobiobox," a high-throughput solution for microalgal screening and culture optimization (Heo et al., 2015). @ Elsevier Ltd. Reproduced with permission.

\section{Measuring Media pH and Dissolved Oxygen}

Finally, a typical commercial bioreactor comes with the option of measuring $\mathrm{pH}$ and oxygen, where the $\mathrm{pH}$ value is usually monitored with an electrochemical probe [a glass or a combination electrode (Bates, 1954; Convinton et al., 1985)]. An alternative that can be integrated into DIY culturing solutions is to use fluorescent $\mathrm{pH}$ and oxygen indicators deposited at the bottom of the culture vessel (Wolfbeis, 1997; John et al., 2003a,b). For example, $\mathrm{pH}$ and dissolved oxygen level can be measured in a microbioreactor using commercially available (Presens $\mathrm{GmbH}$ ) and platinum(II) octaethylporphine-ketone sensor spots (Papkovsky et al., 1995; Lee et al., 2011; Perez-Pinera et al., 2016). Platinum(II) and palladium(II) complexes of the porphyrin ketones exhibit strong phosphorescence that quenches in the presence of oxygen, and the spots can be embedded in polystyrene and immobilized on glass disks (Perez-Pinera et al., 2016).

\section{EXAMPLES}

\section{Assessing Genetic Circuits}

Evaluating the performance of genetic circuits in microplate readers is a common practice, as currently available readers come with OD, fluorescence, and luminescence measurement capabilities, and as methods that deal with auto-fluorescence from the plate and the media, as well as from the cells, have been developed (Boyer et al., 2010; Lichten et al., 2014; Mihalcescu et al., 2015). In combination with robotic liquid handling, throughput reached is sufficiently high to enable measuring of promoter activity on a genomic scale of an entire fluorescent reporter strain library (Zaslaver et al., 2006, 2009). However, commercially available plate readers with (customized) robotic liquid handling capabilities are expensive, and DIY solutions offer low cost and flexible alternative. For example, (Takahashi et al., 2014) used a 3D-printed holder of the culture vessel and a syringe pump from ABS plastic material to design a low cost "turbidostat" (Figure 1a). The device has in-line OD and fluorescence detection capability, implemented via a simple laser diode, light emitting diode (LED), and a photodetector.

\section{Adaptive Laboratory Evolution Experiments}

In laboratory evolution (ALE) experiments microbial cells are cultured for prolonged period of time under a chosen evolutionary pressure, by either serially diluting the culture or in a chemostat mode, Figures 3A,B (Lenski et al., 1991). While such experiments have been performed in laboratories for $\sim 30$ years, recently these are being used for systems and synthetic biology and biotechnology (Buckling et al., 2009; Dragosits and Mattanovich, 2013). Common evolutionary pressures include sublethal antibiotic 


\section{A}

serial dilution
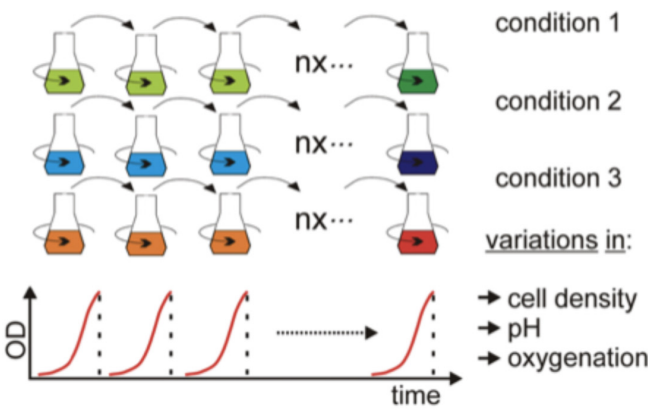

C

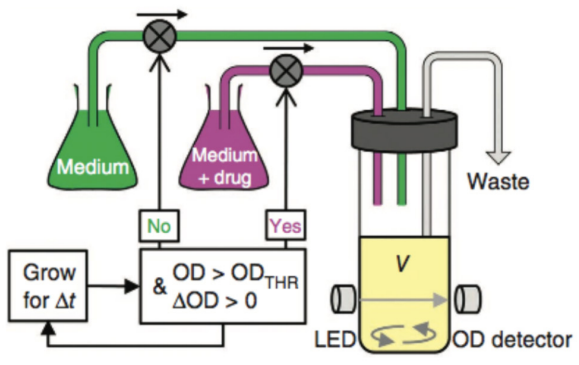

B

chemostat

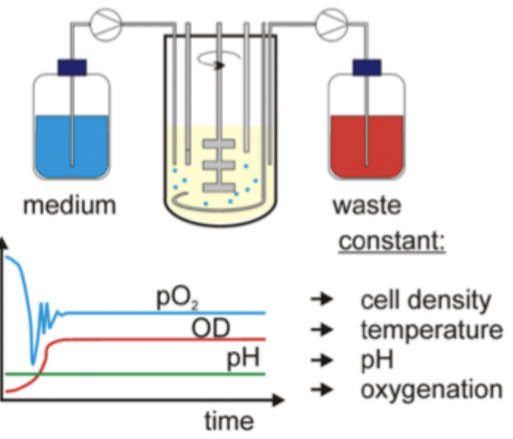

D

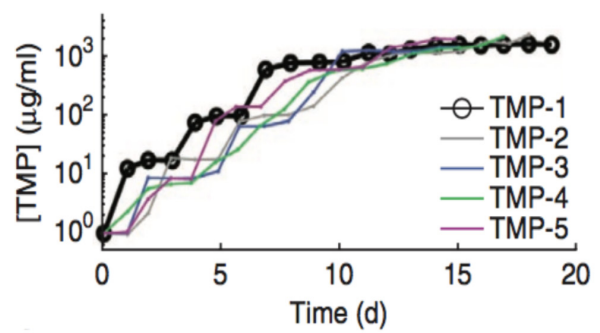

FIGURE 3 | Adaptive laboratory evolution experiment. (A) Serial dilution passage in shaker flasks is often performed manually (Dragosits and Mattanovich, 2013). The growth parameters, such as cell density, $\mathrm{pH}$, and oxygen levels can fluctuate during each growth cycle. () BioMed Central. Reproduced with permission. (B) Chemostat operation (Dragosits and Mattanovich, 2013). The evolution experiment can also be performed with a continuous supply of media and dilution of bacterial cell density. The cell density and other environmental conditions, such as temperature, pH, and oxygen levels are kept constant. @) BioMed Central. Reproduced with permission. (C) A feedback algorithm based on the measured optical density is implemented for dynamic adjustment of the drug concentration in the morbidostat (Toprak et al., 2012). The device operates similar to the chemostat, at a given OD value it dilutes the culture by pumping extra media, except here the additional media contains higher drug concentration with each cycle. () Nature Publishing Group. Reproduced with permission. (D) Representative results from the morbidostat (Toprak et al., 2012). The $\mathrm{IC}_{50}$, defined as the antibiotic inhibitor concentration at which the growth rate is $50 \%$ of the maximal growth at zero inhibitor concentration, is displayed for the drug trimethoprim over the course of 20 days. The legend shows the color codes for five parallel evolution experiments. The stepwise increase can be clearly seen. @ Nature Publishing Group. Reproduced with permission.

concentrations and growth while producing unnecessary proteins or compounds under a given expression system (Dekel and Alon, 2005).

The length of ALE experiments and the need for constant culture dilution, as well as the range of different selection pressures chosen, make them good candidates for automation and DIY design (Gresham and Dunham, 2014). For example, the "morbidostat" is a custom culturing solution that uses a feedback loop to progressively increase antibiotic drug concentration at a given measured $\mathrm{OD}$, and thus keeps the drug-resistant mutant constantly challenged (Toprak et al., 2012, 2013). Using the "morbidostat" and trimethoprim as a test case antibiotic, stepwise increases of antibiotic drug resistance (up to $\sim 1680$ fold for $\sim 20$ days) have been observed and corresponding mutations in the drug's target identified, Figure 3D. Arias Castro JC reports the design of "EvoBot," a robot made from LEGO $^{\circledR}$ MINDSTORMS ${ }^{\circledR}$ NXT 2.0 for automated dilution during ALE experiments (Arias-Castro, 2017). EvoBot is constructed from LEGO Bricks and controlled with RWTH Mindstorms NXT Toolbox for MATLAB (MathWorks). The culture is stirred in a 96-well plate one row at a time, where the robot inoculates every subsequent row and thus reduces the need for human intervention from once a day to approximately once a week.

\section{Optogenetic Intervention Bioreactors}

Recently, a range of monochromatic optogenetic systems has been developed (Levskaya et al., 2005; Ohlendorf et al., 2012; Ryu and Gomelsky, 2014; Schmidl et al., 2014; Ramakrishnan and Tabor, 2016). Additionally, two-color control has been achieved (Tabor et al., 2011) and recently, three-color RGB vision has been demonstrated in E. coli (Fernandez-Rodriguez et al., 2017).

Light is a non-contact and versatile solution for bioprocess control and as such has been implemented in several custom culturing devices and as an add-on option to commercially available plate readers. For example, (Davidson et al., 2013) developed an array of LEDs that can be fitted to the bottom of a microplate for optogenetic control, and Tabor and colleagues constructed a Light Tube Array and Light Plate Apparatus to deliver light to individual wells on a multiwell plate (Olson et al., 2014; Gerhardt et al., 2016). The versatility that can be achieved allowed (Davidson et al., 2013) to show that a specific two-component system from cyanobacteria acts as a low pass filter when expressed in E. coli 
and (Gerhardt et al., 2016) successfully programmed specific gene expression profiles. Similarly, several groups have reported custom designed light-controlled bioreactors, using either multiwell plates (Lee et al., 2013) or larger volume vessels (MiliasArgeitis et al., 2011; Ruess et al., 2015; Wang and Yang, 2017), demonstrating switchable control of gene expression in microorganisms.

Add-on solutions to achieving optogenetic control have also been reported. For example, Moglich and colleagues modified an existing Tecan microplate reader and added optical fiber for light illumination (Richter et al., 2015; Figure 2a). The system has been used for in vitro study of Arabidopsis thaliana phytochrome $B$ photoactivation, but can, in principle, be used for optogenetic control.

\section{Photobioreactor for Photosynthetic Microalgae}

Microalgae are an important feedstock for production of useful compounds, such as biofuels and high-value chemicals (Chisti, 2007; Angermayr et al., 2015). Furthermore, their ability to capture $\mathrm{CO}_{2}$ while producing biomass, which in turn is a source of energy and bioproducts, has gained them increasing attention (Katiyar et al., 2017). However, microalgae are highly diverse with an estimated 200,000-1,000,000 existing species (Guiry, 2012), with each species requiring different light intensity, temperature, and carbon levels. Thus, optimizing cultivation conditions for the screening of potentially useful algae is a research challenge that can benefit from the flexibility and low cost of DIY solutions. For example, Chen and colleagues assembled optical microplates, using LEDs to supply light to individual wells and study lipid conversion efficiency of Dunaliella tertiolecta.
Similarly, the recently developed "Photobiobox" integrates 96well plate with LEDs for illumination, coupled with light gradient filters, and water blocks to achieve light intensity and temperature gradients (Heo et al., 2015), Figure 2c. The device was used to screen 12 microalgae strains from fresh water for their growth and lipid production potential. Add-on solutions have also been proposed, Morschett and colleagues modified a commercial microplate reader for 48fold parallelized algae cultivation by incorporating a "photo module" for individual well illumination (Morschett et al., 2017).

\section{Anaerobic Cultivation}

Anaerobic cultivation of microorganism is of increasing interest in industrial biotechnology, e.g., bacterium Clostridium that biochemically synthesizes solvents (Green, 2011). Most commonly, anaerobic cultivation is achieved with the use of specialized anaerobic cabinets (Leach et al., 1971). While agar plate growth can be performed in anaerobic jars (Brewer and Brown, 1938; Brewer, 1942) and gas packs, and commercially available tubes with rubber stoppers can be used for liquid culture growth (Anderson and Fung, 1983), using these without the anaerobic cabinets makes it difficult to monitor culture growth (for example, measure OD). We envision that DIY culturing technology can simplify anaerobic cultivation and enable cheap and innovative solutions. For example, recently reported Moorella thermoacetica that can self-photosynthesize via synthetically introduced cadium sulfur (CdS) nanoparticles, is strictly an anaerobe (Drake and Daniel, 2004; Sakimoto et al., 2016). Thus, the reported photosynthesis requires cultivation under anaerobic conditions in the presence of light. DIY

TABLE 1 | Summary of DIY bioreactor and microplates.

\begin{tabular}{|c|c|c|c|c|c|}
\hline Name & In-line detection & Volume & Organism & Note & Reference \\
\hline Turbidostat & OD, fluorescence & $15 \mathrm{ml}$ & Yeast & 3D printed holder and pump & Takahashi et al., 2014 \\
\hline Morbidostat & OD & $12 \mathrm{ml}$ & E. coli & Antibiotic drug resistance & $\begin{array}{l}\text { Toprak et al., 2012, } \\
\text { 2013; Liu et al., } 2016\end{array}$ \\
\hline Microreactor & $\begin{array}{l}\mathrm{OD}, \mathrm{PH}, \text { and } \\
\text { oxygen }\end{array}$ & $1 \mathrm{ml}$ & E. coli & Point of care & $\begin{array}{l}\text { Lee et al., 2011; } \\
\text { Perez-Pinera et al., } \\
2016\end{array}$ \\
\hline Optogenetic bioreactor & OD fluorescence & $12 \mathrm{ml}$ & E. coli & Optogenetics & Wang and Yang, 2017 \\
\hline Light tube array (LTA) & $\begin{array}{l}\text { None (fluorescence } \\
\text { with offline flow } \\
\text { cytometry) }\end{array}$ & $1 \mathrm{ml}$ & E. coli & $\begin{array}{l}\text { Optogenetics for bacteria and } \\
\text { mammalian cell entrapment of } \\
\text { cyanobacteria }\end{array}$ & $\begin{array}{l}\text { Olson et al., 2014; } \\
\text { Gerhardt et al., } 2016\end{array}$ \\
\hline Cell growth quantifier (CGQ) & OD & $50 \mathrm{ml}$ & Yeast & $\begin{array}{l}\text { Back scattering and high speed } \\
\text { data acquisition }\end{array}$ & Bruder et al., 2016 \\
\hline Microplate with robotic assay & OD, fluorescence & $\sim 100 \mu \mathrm{l}$ & E. coli & Gene expression profiling & $\begin{array}{l}\text { Zaslaver et al., 2006, } \\
\text { 2009; Huber et al., } \\
2009\end{array}$ \\
\hline Optical Microplate & $\begin{array}{l}\text { None (fluorescence } \\
\text { with offline flow } \\
\text { cytometry) }\end{array}$ & $100 \mathrm{ml}$ & $\begin{array}{l}\text { E. coli } \\
\text { Dunaliella } \\
\text { tertiolecta }\end{array}$ & Photosynthesis optogenetics & $\begin{array}{l}\text { Chen et al., 2012; } \\
\text { Davidson et al., 2013; } \\
\text { Lee et al., } 2013\end{array}$ \\
\hline PhotobioBox & OD & $200 \mathrm{ml}$ & Microalgae & $\begin{array}{l}\text { Light intensity and temperature } \\
\text { gradient between the wells }\end{array}$ & Heo et al., 2015 \\
\hline
\end{tabular}

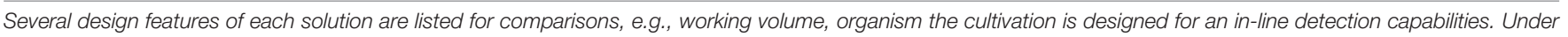
the "Note" column we have listed the main intended application or any special features of the solution. 
solutions could be designed to fit the purpose, e.g., bioreactors can be furnished with LED light and specific gas source. Low power electronic components such as LEDs and semiconductor photodetectors can run on battery power, and transmission of data collected can be achieved with wireless communication, which leaves the possibility of designing solutions that can be placed in specialized environments as well. We, therefore, believe DIY solution for anaerobic growth can widen the extent and ease with which such microorganisms are cultured.

\section{CONCLUSION}

We have surveyed some of the DIY solutions for microbial cultivation as summarized in Table 1. While no single solution satisfies all the requirements of different research communities, our intention was to demonstrate that DIY solutions are becoming ever more cost-effective and user-friendly and can expand the range of experiments that can be performed in a given laboratory. We would also like to draw the reader's attention

\section{REFERENCES}

Anderson, K. L., and Fung, D. Y. C. (1983). Anaerobic methods, techniques and principles for food bacteriology: a review. J. Food Prot. 46, 811-822. doi: 10.4315/0362-028X-46.9.811

Angermayr, S. A., Rovira, A. G., and Hellingwerf, K. J. (2015). Metabolic engineering of cyanobacteria for the synthesis of commodity product. Trends Biotechnol. 33, 352-361. doi: 10.1016/j.tibtech.2015. 03.009

Arias-Castro, J. C. (2017). Physiology, Evolution And Single Cell Gene Expression: An Experimental Approach Using A Microfluidics Technique, Ph.D. thesis, Universidad de los Andes, Bogota.

Baden, T., Chagas, A., Gage, G., Marzullo, T., Prieto Godino, L., and Euler, T. (2015). Open labware-3D printing your own lab equipment. PLoS Biol. 13:e1002175. doi: 10.1371/journal.pbio.1002175

Bates, R. G. (1954). Determination of Ph: Theory and Practice. Hoboken, NJ: Wiley. Betts, J., and Baganz, F. (2006). Miniature bioreactors: current practices and future opportunities. Microb. Cell Fact. 5:21 doi: 10.1186/1475-2859-5-21

Boyer, F., Besson, B., Baptist, G., Izard, J., Pinel, C., Ropers, D., et al. (2010). WellReader: a MATLAB program for the analysis of fluorescence and luminescence reporter gene data. Bioinformatics 26, 1262-1263 doi: 10.1093/ bioinformatics/btq016

Brewer, J. H. (1942). A new petri dish cover and technique for use in the cultivation of anaerobes and microaerophiles. Science 95:987. doi: 10.1126/science.95. 2475.587

Brewer, J. H., and Brown, J. H. (1938). A method for utilizing illuminating gas in the brown, fildes and McIntosh or other anaerobic jars of the Laidlaw principle. J. Lab. Clin. Med. 23, 870-874.

Bruder, S., Reifenrath, M., Tomik, T., Boles, E., and Herzog, K. (2016). Parallelised online biomass monitoring in shake flasks enables efficient strain and carbon source dependent growth characteisation of Saccharomyces cerevisiae. Microb. Cell Fact. 12:127 doi: 10.1186/s12934-016-0526-3

Buckling, A., Craig Maclean, R., Brockhurst, M. A., and Colegrave, N. (2009). The beagle in a bottle. Nature 457, 824-829 doi: 10.1038/nature07892

Chen, M., Taulant, M., Hollanda, T., and Basu, A. S. (2012). Optical microplates for high-throughput screening of photosynthesis in lipid-producing algae. $L a b$ Chip 12, 3870-3874 doi: 10.1039/c2lc40478h.

Chisti, Y. (2007). Biodiesel from microalgae. Biotechnol. Adv. 25, 294-306. doi: 10.1016/j.biotechadv.2007.02.001

Convinton, A. K., Bates, R. G., and Durst, R. A. (1985). Definition of pH scales, standard reference values, and related terminology. Pure Appl. Chem. 57, 531-542. doi: 10.1351/pac198557030531 to the "Open-Labware" collections that are aiming to bring together different DIY projects beyond, but including, cultivation technologies (Baden et al., 2015). We anticipate this community to grow and offer further improved and increasingly versatile designs.

\section{AUTHOR CONTRIBUTIONS}

Y-TY conceived the original idea. TP and Y-TY wrote the manuscript.

\section{FUNDING}

Y-TY would like to acknowledge funding support from the Ministry of Science and Technology under grant number MOST-105-2221-E-007-130-MY3. TP has been supported by the CBMNet Grant BB/L013703/1 and the BBSRC/EPSRC/MRC Synthetic Biology Research Centre grant (BB/M018040/1).

Davidson, E. A., Basu, A. S., and Bayer, T. S. (2013). Programming microbes using pulse width modulation of optical signals. J. Mol. Biol. 425, 4161-4166. doi: 10.1016/j.jmb.2013.07.036

Dekel, E., and Alon, U. (2005). Optimality and evolutionary tuning of the expression level of a protein. Nature 436, 588-592. doi: 10.1038/nature0 3842

Dragosits, M., and Mattanovich, D. (2013). Adaptive laboratory evolution principles and applications for biotechnology. Microb. Cell Fact. 12:64. doi: 10.1186/1475-2859-12-64

Drake, H. L., and Daniel, S. L. (2004). Physiology of the thermophilic acetogen Moorella thermoacetica. Res. Microbiol. 155, 869-883. doi: 10.1016/j.resmic. 2004.10.002

Fernandez-Rodriguez, J., Moser, F., Song, M., and Voigt, C. A. (2017). Engineering RGB color vision into Escherichia coli. Nat. Chem. Biol. 13, 706-710. doi: 10. 1038/ncmebio. 2390

Ferry, M. S., Razinkov, I. A., and Hasty, J. (2011). Methods in Enzymology, Vol. 497, 2011th Edn. Amsterdam: Elsevier.

Gerhardt, K. P., Olson, E. J., Castillo-Hair, S. M., Hartsough, L. A., Landry, B. P., Ekness, F., et al. (2016). An open-hardware platform for optogenetics and photobiology. Sci. Rep. 6:35363. doi: 10.1038/srep35363

Green, E. M. (2011). Fermentative production of butanol - the industrial perspective. Curr. Opin. Biotechnol. 22, 337-343 doi: 10.1016/j.copbio.2011. 02.004

Gresham, D., and Dunham, M. J. (2014). The enduring utility of continuous culturing in experimental evolution. Genomics 104, 399-405. doi: 10.1016/j. ygeno.2014.09.015

Guiry, M. D. (2012). How many species of algae are there? J. Phycol. 48, 1057-1063. doi: 10.1111/j.1529-8817.2012.01222.x

Gupta, A., and Rao, G. (2003). A study of oxygen transfer in shake flask using a non-invasive oxygen sensor. Biotechnol. Bioeng. 5, 351-358. doi: 10.1002/bit. 10740

Han, A., Hou, H., Li, L., Kim, H. S., and de Figueiredo, P. (2013). Microfabricated devices in microbial bioenergy sciences. Trends Biotechnol. 31, 225-232. doi: 10.1016/j.tibtech.2012.12.002

Hegab, H. M., Elmekawy, A., and Stakenborg, T. (2013). Review of microfluidic microbioreactor technology for high-throughput submerged microbiological cultivation. Biomicrofluidics 7:21502. doi: 10.1063/1.479 9966

Heo, J., Cho, D. H., Ramanan, R., Oh, H. M., and Kim, H. S. (2015). PhotoBiobox: a tablet sized, low-cost, high throughput photobioreactor for microalgal screening and culture. Biochem. Eng. J. 103, 193-197. doi: 10.1016/j.bej.2015. 07.013 
Hermann, R., Lehmann, M., and Büch, J. (2003). Characterization of gas-liquid mass transfer phenomena in microtiter plates. Biotechnol. Bioeng. 81, 178-186. doi: 10.1002/bit.10456

Huber, R., Ritter, D., Hering, T., Hillmer, A. K., Kensy, F., Müller, C., et al. (2009). Robo-Lector - a novel platform for automated high-throughput cultivations in microtiter plates with high information content. Microb. Cell Fact. 8:42. doi: 10.1186/1475-2859-8-42

Jepson, A. K. (2014). E. Coli Motility and Growth: A Biophysical Study. Ph.D. thesis, University of Edinburgh, Edinburgh.

John, G. T., Goelling, D., Klimant, I., Schneider, H, and Heinzle, E. (2003a). pHsensing 96-well microtiter plates for the characterization of acid production by dairy starter cultures. J. Dairy Res. 70, 327-333.

John, G. T., Klimant, I., Wittmann, C, and Heinzle, E. (2003b). Integrated optical sensing of dissolved oxygen in microtiter plates: a novel tool for microbial cultivation. Biotechnol. Bioeng. 81, 829-836

Katiyar, R., Gurjar, B. R., Biswas, S., and Kumar, P. (2017). Microalgae: an emerging source of energy based bio-products and a solution for environmental issues. Renew. Sustain. Energy Rev. 72, 1083-1093. doi: 10.1016/j.rser.2016.10.028

Kishony, R., and Leibler, S. (2003). Environmental stresses can alleviate the average deleterious effect of mutations. J. Biol. 2:14.

Klöckner, W., and Büchs, J. (2012). Advances in shaking technologies. Trend Biotechnol. 30, 307-314. doi: 10.1016/j.tibtech.2012.03.001

Koch, A. L. (1970). Turbidity measurements of bacterial cultures in some available commercial instruments. Anal. Biochem. 38, 252-259 doi: 10.1016/00032697(70)90174-0

Leach, P. A., Bullen, J. J., and Grant, I. D. (1971). Anaerobic $\mathrm{CO}_{2}$ cabinet for the cultivation of strict anaerobes. Appl. Microbiol. 22, 824-827.

Lee, J. M., Lee, J., Kim, T., and Lee, S. K. (2013). Switchable gene expression in Escherichia coli using a miniaturized photobioreactor. PLoS One 8:e52382. doi: 10.1371/journal.pone.0052382

Lee, K. S., Boccazzi, P., Sinskey, A. J., and Ram, R. J. (2011). Microfluidic chemostat and turbidostat with flow rate, oxygen, and temperature control for dynamic continuous culture. Lab Chip 11, 1730-1739. doi: 10.1039/c1lc20019d

Lenski, R. E., Rose, M. R., Simpson, S. C., and Tadler, S. C. (1991). Long-term experimental evolution in Escherichia coli. I. Adaptation, and divergence during 2,000 generations. Am. Nat. 138, 1315-1341. doi: 10.1086/285289

Levskaya, A., Chevalier, A. A., Tabor, J. J., Simpson, Z. B., Lavery, L. A., Levy, M., et al. (2005). Synthetic biology: engineering Escherichia coli to see light. Nature 438, 441-442. doi: 10.1038/nature04405

Lichten, C. A., White, R., Clark, I. B., and Swain, P. S. (2014). Unmixing of fluorescence spectra to resolve quantitative time-series measurements of gene expression in plate readers. BMC Biotechnol. 14:11. doi: 10.1186/1472-675014-11

Liu, P. C., Lee, Y. T., Wang, C. Y., and Yang, Y. T. (2016). Design and use of a low cost, automated morbidostat for adaptive evolution of bacteria under antibiotic drug selection. J. Vis. Exp. 1154:e54426. doi: 10.3791/54426

Mihalcescu, I, Van-Melle Gateau, M, Chelli, B, Pinel, C, Ravanat, J. L. (2015). Green autofluorescence, a double edged monitoring tool for bacterial growth and activity in micro-plates. Phys. Biol. 12:066016 doi: 10.1088/1478-3975/12/ $6 / 066016$

Milias-Argeitis, A., Summers, S., Stewart-Ornstein, J., Zuleta, I., Pincus, D., El-Samad, H., et al. (2011). In silico feedback for in vivo regulation of a gene expression circuit. Nat. Biotechnol. 29, 1114-1116 doi: 10.1038/nbt. 2018

Morschett, H., Schiprowski, D., Müller, C. Mertens, K. Felden, P., Meyer, J., et al. (2017). Design and validation of a parallelized micro-photobioreactor enabling phototrophic bioprocess development at elevated throughput. Biotechnol. Bioeng. 114, 122-131. doi: 10.1002/bit.26051

Novick, A., and Szilard, L. (1950). Description of chemostat. Science 112, 715-716. doi: 10.1126/science.112.2920.715

Ohlendorf, R., Vidavski, R. R., Eldar, A., Moffat, K., and Möglich, A. (2012). From dusk till dawn: one-plasmid systems for light-regulated gene expression. J. Mol. Biol. 416, 534-542. doi: 10.1016/j.jmb.2012.01.001

Olson, E. J., Hartsough, L. A., Landry, B. P., Shroff, R., and Tabor, J. J. (2014). Characterizing bacterial gene circuit dynamics with optically programmed gene expression signals. Nat. Methods 11, 449-455. doi: 10.1038/nmeth.2884

Papkovsky, D. B., Ponomarev, G. V., Trettnak, W., and O'Leary, P. (1995). Phosphorescent complexes of porphyrin ketones: optical properties and application to oxygen sensing. Anal. Chem. 67, 4112-4117, doi: 10.1021/ ac00118a013

Perez-Pinera, P., Han, N., Cleto, S., Cao, J., Purcell, O., Shah, K. A., et al. (2016). Synthetic biology and microbioreactor platforms for programmable production of biologics at the point of care. Nat. Commun. 7:12211. doi: $10.1038 /$ ncomms 12211

Pleiss, J. (2006). The promise of synthetic biology. Appl. Microbiol. Biotechnol. 73, 735-739. doi: 10.1007/s00253-006-0664-3

Ramakrishnan, P., and Tabor, J. J. (2016). Repurposing Synechocystis PCC6803 UirS-UirR as a UV-violet/green photoreversible transcriptional regulatory tool in E. coli. ACS Synth. Biol. 5, 733-740. doi: 10.1021/acssynbio.6b0 0068

Richter, F., Scheib, U. S., Mehlhorn, J., Schubert, R., Wietek, J., and Gernetzki, O., et al. (2015). Upgrading a microplate reader for photobiology and all-optical experiments. Photochem. Photobiol. Sci. 14, 270-279. doi: 10.1039/c4pp0 $0361 \mathrm{f}$

Ruess, J., Parise, F., Milias-Argeitis, A., Khammash, M., and Lygerosa, J. (2015). Iterative experiment design guides the characterization of a light-inducible gene expression circuit. Proc. Natl. Acad. Sci. U.S.A. 112, 8148-8153. doi: 10.1073/ pnas. 1423947112

Ryu, M. H., and Gomelsky, M. (2014). Near-infrared light responsive synthetic c-di-GMP module for optogenetic applications. ACS Synth. Biol. 3, 802-810. doi: $10.1021 / \mathrm{sb} 400182 \mathrm{x}$

Sakimoto, K. K., Wong, A. B., and Yang, P. (2016). Self-photosensitization of nonphotosynthetic bacteria for solar-to-chemical production. Science 351, 74-77. doi: 10.1126/science.aad3317

Schmidl, S. R., Sheth, R. U., Wu, A., and Tabor, J. J. (2014). Refactoring and optimization of light-switchable Escherichia coli two-component systems. ACS Synth. Biol. 3, 820-831. doi: 10.1021/sb500273n

Schmidt-Hager, J., Ude, C., Findeis, M., John, G. T., Scheper, T., Beutel, S. (2014). Noninvasive online biomass detector system for cultivation in shake lasks. Eng. Life Sci. 14, 467-476. doi: 10.1002/elsc.201400026

Scognamiglio, V., Antonacci, A., Lambreva, M. D., Litescu, S. C., and Rea, G. (2015). Synthetic biology and biomimetic chemistry as converging technologies fostering a new generation of smart biosensors. Biosens. Bioelectron. 74, 1076-1086. doi: 10.1016/j.bios.2015.07.078

Sklodowska, K., and Jakiela, S. (2017). Enhancement of bacterial growth with the help of immiscible oxygenated oils. RSC Adv. 7, 40990-40995. doi: 10.1039/ c7ra07095k

Stevenson, K. F., McVey, A. F., Clark, I. B. N., Swain, P. S., and Pilizota, T. (2016). General calibration of microbial growth in microplate readers. Sci. Rep. 6:38828. doi: $10.1038 /$ srep38828

Tabor, J. J., Levskaya, A., and Voigt, C. A. (2011). Multichromatic control of gene expression in Escherichia coli. J. Mol. Biol. 405, 315-324 doi: 10.1016/j.jmb.2010. 10.038

Takahashi, C. N., Miller, A. W., Ekness, F., Dunham, M. J., and Klavins, E. (2014). A low cost, customizable turbidostat for use in synthetic circuit characterization. ACS Synth. Biol. 4, 32-38. doi: 10.1021/sb500165g

Toprak, E., Veres, A., Michel, J. B., Chait, R., Hartl, D. L., and Kishony, R. (2012). Evolutionary paths to antibiotic resistance under dynamically sustained drug selection. Nat. Genet. 44, 101-106. doi: 10.1038/ng.1034

Toprak, E., Veres, A., Yildiz, S., Pedraza, J. M., Chait, R., Paulsson, J., et al. (2013). Building a morbidostat: an automated continuous-culture device for studying bacterial drug resistance under dynamically sustained drug inhibition. Nat. Protoc. 8, 555-567. doi: 10.1038/nprot.nprot.2013.021

Wang, H. K., and Yang, Y. T. (2017). Mini photobioreactors for in vivo real-time characterization and evolutionary tuning of bacterial optogenetic circuit. ACS Synth. Biol. 6, 1793-1796. doi: 10.1021/acssynbio.7b0009

Winson, M. K., Swift, S., Hill, P. J., Sims, C. M., Griesmayr, G., Bycroft, B. W., et al. (1998). Engineering the luxCDABE genes from Photorhabdus luminescens to provide a bioluminescent reporter for constitutive and promoter probe plasmids and mini-Tn5 constructs. FEMS Microbiol. Lett. 163, 193-202. doi: 10.1111/j.1574-6968.1998.tb13045.x

Wolfbeis, O. S. (1997). “Chemical sensing using indicator dyes", in Optical Fiber Sensors 4. Applications, Analysis, and Future Trends, eds J. Dakin and B. Culshaw (London: Artech House), 53-107.

Yang, Y. T., and Wang, C. Y. (2016). Review of microfluidic photobioreactor technology for metabolic engineering and synthetic biology of 
cyanobacteria and microalgae. Micromachines 7, 185-202. doi: 10.3990/mi71 00185

Yeh, P., Tsehumi, A., and Kishony, R. (2006). Functional classification of drugs by properties of their pairwise interactions. Nat. Gen. 38, 489-494. doi: 10.10138/ ng1755

Zaslaver, A., Bren, A., Ronen, M., Itzkovitz, S., Kikoin, I., Shavit S., et al. (2006). A comprehensive library of fluorescent transcriptional reporters for Escherichia coli. Nat. Methods 3, 623-628. doi: 10.1038/nmeth895

Zaslaver, A., Kaplan, S., Bren, A., Jinich, A., Mayo, A., Dekel, E., et al. (2009). Invariant distribution of promoter activities in Escherichia coli. PLoS Comput. Biol. 5:e1000545. doi: 10.1371/journal.pcbi.10 00545
Conflict of Interest Statement: The authors declare that the research was conducted in the absence of any commercial or financial relationships that could be construed as a potential conflict of interest.

The reviewer PS and handling Editor declared their shared affiliation.

Copyright (c) 2018 Pilizota and Yang. This is an open-access article distributed under the terms of the Creative Commons Attribution License (CC BY). The use, distribution or reproduction in other forums is permitted, provided the original author(s) and the copyright owner(s) are credited and that the original publication in this journal is cited, in accordance with accepted academic practice. No use, distribution or reproduction is permitted which does not comply with these terms. 